\title{
Late Life Depression: Review of Perception, Assessment and Management in Community Dwellers
}

\author{
Olabisi Modupe Osimade* and Jonathan Osiki \\ $\mathrm{PhD}$ (Clinical Psychology), University of Ibadan, Nigeria
}

*Corresponding author: Dr. Olabisi Modupe Osimade, PhD (Clinical Psychology), University of Ibadan, Nigeria; Tel: +2348164060420; Email: osimadeolabisi@yahoo.com

Received: February 04, 2022; Accepted: February 10, 2022; Published: February 11, 2022

\begin{abstract}
Depression is one of the mental health conditions identified and projected to be the second leading cause of burden of disease in late life and unfortunately concerns for its debilitating consequences have birthed the need for continuous studies into its etiology, risk factors and management options (WHO, 2017). As the world aged population increases (by 2 billion persons in the next 30 years from 7.7 billion currently to 9.7 billion in 2050), number of older adults aged 65 years and over is projected to proportionately grow by 50\% from about 727 million in 2020 to more than 1.5 billion by 2050 (United Nations, 2020) invariably implying that one in six people worldwide will be aged 65 years and over. Averagely, the number of older adults manifesting symptoms of mental disorders such as depression is set to increase proportionately among the estimated population.

The sudden but precipitated increase in incidences of depression across all ages had necessitated the need for constant reviews of mental health disorders. Factors that informed the mental health decisions of community dwelling older adults are hinged on their personal convictions on the presence of the disease and not just another somatic related illness. These convictions are quite germane to the kind of treatment they sought for and could invariably be the major reason why geriatric depression has consistently been misdiagnosed or undertreated very often among community dwelling older adults.
\end{abstract}

Keywords: Late life depression, Mental health, Burden of disease, Community dwelling older adults

\section{Introduction}

Depression is one of the mental health conditions identified and projected to be the second leading cause of burden of disease in late life and unfortunately concerns for its debilitating consequences have birthed the need for continuous studies into its etiology, risk factors and management options across all populations and samples [1]. As the world aged population increases (by 2 billion persons in the next 30 years from 7.7 billion currently to 9.7 billion in 2050), number of older adults aged 65 years and over is projected to proportionately grow by $50 \%$ from about 727 million in 2020 to more than 1.5 billion by 2050 [2] invariably implying that one in six people worldwide will be aged 65 years and over. Averagely, the number of older adults manifesting symptoms of mental disorders such as depression is set to increase proportionately among the estimated population. Good news however is that mental health issues in late life has been explored within different population of older adults globally.

Specifically, literature exploring late life depression in community samples and in clinical trials have been widely explored [3-7]. Though comparison in case reports from continents varies as limited number of accounts of depression among older adults in Africa constitute a major setback in reports and management [7]. Despite the disparities in reports and studies conducted all over the world, the goal of research in bridging gaps in findings had been successfully achieved; studies had established the etiology of late life depression, consequences of untreated depression and management options had also been widely appraised. This review will address types of depression relative to older adults, diagnosis and complications of undiagnosed late life depression, the interpretation and assessment of depressive symptoms and experience of depression in some Nigerian communities will also be explored. Publications on the psychological management and implications of culture on the expression of depression in community samples of older adults will also be appraised in relation to their clinical and research implications.

\section{Late Life Depression}

Psychological issues have remained an issue met with lots of reluctance and ignorance which older adults are always embarrassed to describe. Presentation of depression in late life which often include insomnia (complaints about difficulties in recall and identification), social withdrawal (lack of interest in social activities and behavioural changes characteristic of hostility (irritability), frequent unexplained falls, hallucination, agitation etc. are often missed for age-related illnesses or somatic complaints [8]. The Diagnostic and Statistical Manual of Mental Disorders (DSM5), gave a clear description of Major Depressive Disorder (MDD) as: "a manifestation of multiple major depressive fits". A depressive fit is described in the DSM-5 as mood change characterized by variations in emotions resulting in a continuous manifestation of multiple characteristics of depressive behaviours usually exhibited concurrently for two weeks. Other accompanying symptoms may include daily display of a characteristically low emotional state 
observed either by the sufferer or observation made by others [9]. Anhedonia, described as an identifiable attention shift from all or from about all activities of choice, spanning throughout the day or on daily basis. Drastic loss or gain of body weight, daily amnesia or hypermnesia [10], daily mental degeneration, daily burnout (feeling stressed), excessive or inappropriate self-attribution of guilt and or cognitive retardation [11].

There must be an accompanying diagnosable distress or discomfort and social withdrawal following the listed symptoms to meet the criteria of the DSM-5. Of the five symptoms, the sufferer must either have a "depressed mood" or totally lose interest in pleasure or social activities. Other symptoms typical of late life depression include somatic complaints, cognitive impairment, persistent anhedonia or loss of pleasure, behavior changes and the pronounced presentation of negative personality traits $[12,13]$. There has been an age long societal misconception about aging and its accompanying misery. Coincidentally, this reflection has been documented in literature, myths and beliefs of people across diverse communities all over the world [9]. [14] in his argument for the inclusion of counseling sessions in the proper diagnosis and treatment of age related physical and mental illnesses established that many physical manifestations of late life depression could mimic the symptoms of illnesses peculiar with old age making depression diagnosis relatively difficult [10].

\section{Depressive Disorders in Late Life}

\section{Major Depression}

Major depression, also referred to as depression is a mental health disorder characterized by at least two weeks of consistent negative mood swings observed across the individual's activities of daily living. It is characterized by poor self-esteem, loss of interest in normally enjoyable activities, fatigue, and bodily ache [15]. There is a close similarity between the DSM-IV-TR [16] criteria for major depression in both old and younger individuals and the DSM-5. Major depression in older adults may be preceded by cognitive impairment that may develop after the onset of depression, this condition is described in [17] as dementia syndrome of depression, because of the obvious cognitive deficits.

\section{Vascular Depression}

Vascular depression is the comorbid presence of heart related complications in depressed older adults diagnosed with their first onset of depression [18]. There is the hypothesis that manifestation of late life depression with associating vascular risk factors is complicating to both the sufferer and the clinicians. The risks of misdiagnosis for clinicians are higher, medication interference, and nihilistic attitude from the patient are all compounding for vascular depressive patients [19].

\section{Psychotic Depression}

Clinically diagnosed psychotic depression is any form of depression associated with physical characteristics like hallucination, delusions and violent agitation. Psychotic depression is most common in late life; it includes many difficult to treat symptoms like hallucinations, delusions paranoia, and weight loss due to not eating and dehydration due to not drinking [20].

\section{Dysthymia}

Late onset of dysthymia in late life have a higher prevalence and comorbid factor of cardiovascular disease but are otherwise similar to older patients with late onset depression [21]. Dysthymia is a psychological disorder with presenting symptoms closely mimicking depression with a repeated occurrence lasting through several days or years. Dysthymia is associated with the risk of the development of major depression in older adults, so-called "double-depression," and may be particularly treatment resistant [18].

\section{Diagnosis and Complications Late Life Depression}

Depression assessmentforolderadultscanbechallenging, especially in the physically frail and cognitively impaired. Coexisting medical conditions typical with old age can increase older adult's susceptibility to depression [22]. Proper assessment to rule out the possibilities of other physical health conditions such as hypothyroidism, alcohol use, incontinence, falls, partial and total stroke, cardiac related issues, drug abuse etc. that may contribute to depression need be done before the major depressive disorder diagnosis [23]. Researchers and caregivers are daily challenged on the choice of assessment techniques, diagnosis and identification of depression pointers. For clinicians working with older adults, identifying the prognostic consequences of comorbid medical illnesses in geriatric depression is essential to management and treatment planning [20]. These accompanying medical illnesses poses great difficulties in diagnosing depression having established the interference of medical conditions overlapping affective disorder symptoms. Clinicians must attain a balance when assessing older adults for depression between assumptions [24] (misunderstanding the psychomotor decline in Parkinson disease for a depressive disorder) and outrightly exclusive (erroneously dismissing an older adult's mood swings as "understandable" and typical with old age).

Additionally, for the geriatric population, a number of medications are believed to have interactive effect on their emotions and psychological dispositions thereby igniting a depressive disorder. Use of prescribed medications in old age if not religiously taken may have associating consequences [10]. Depression is a state of emotional disequilibrium which can be managed with clinically prescribed antidepressants. Antidepressant use is known to have a higher interference effects with other medications prescribed for use in old age. [25] reported that sampled cases, market survey reports and reflective studies have carefully examined the relative effects of medications like antipsychotics, corticosteroids, endocrine-altering medications, stimulants etc. with late life depression. Other factors hindering proper diagnosis of geriatric depression may include poor and incoherent communication skills in the elderly [24], presence of multiple somatic complaints not well expressed (Isabella \& Henrietta, 2008), time constraints during clinical visits among others.

Geriatric depression left untreated or undiagnosed predisposes older adults to chronic effects of the disorder. Complications of untreated geriatric depression identified in literature may include: 


\section{Worsening Emotional Well Being}

Old age is presumably a time when older adults are assumed to observe lots of fun activities to relieve already suppressed emotions. It is presumed to be a time to reminiscence and relish the accompanying relief of leaving an active phase of life. Unfortunately, aging and its accompanying complexities are not always perfect as assumed. Sporadic turn of events accompanying physical frailty, systemic degeneration, osteoporosis, incontinence etc. are not in totality very tolerable, they tend to weigh heavily on older adult's psychological disposition [27]. Geriatric depression is a psychological disorder that requires a balance in cognition, attitude and genetic composition. Older adult's inability to maintain a balanced emotion are at a greater risk of having a depressive episode with its associating risk factors [28].

\section{Suicidal Ideation, Act and Behaviour}

Suicide is unarguably the commonest and an extreme psychiatric emergency [29]. The World Health Organization (2000) definition of suicide as a self-attempt to kill and deliberately terminate life initiated and orchestrated by the individual having a full knowledge of the consequences and fatality is just a total summation of the act and its consequence. [30] in their documentation of the incidence and prevalence rates of suicide in the geriatric population presented a figure that is non-comparable with the younger population across most countries. Expressively implying that older adults are at a greater risk of death by self-suicide than the younger population. For every suicide attempted, the margin of those successfully completed is higher in the geriatric population as with a suicide reported in about twelve attempts, four will be effectively completed [31]. In other words, suicide attempts among the geriatric population should be seen as a serious health concern.

Reports on suicide and suicidal attempts in Nigeria taking instances from the stories of an unidentified medical doctor's suicide on the Third Mainland Bridge in Lagos reported by [32], the story of a final year student of Ladoke Akintola University of Technology, Ogbomosho, with a 'First Class' who died of self-poisoning, a woman reported to have been rescued from suicidal attempt on the same mainland bridge who confessed to hearing voices calling her to come to the bridge and jump etc. The disappointing part of these stories which foregrounds the difficulty in tackling depression-induced deaths was the comment of an unnamed friend of the medical doctor whose case seemed to be most recent, about the deceased being very funny and lively [24]. In advanced stage of depression especially in older adults, they cover up with laughter and tend to be extroverted when around people so as to hide deeper psychological 'injury'. Sadly, this type of comment follows virtually every case of suicide reported in Nigeria, [32]. Psycho-pathologically, in a deeply cultural and religious Nigerian society where awkward death by suicide are never taken as a possible negative response of mental disorders, it is not surprising to see people attributing suicide to witchcraft. While the possibility of such a cause cannot be entirely ruled out due to the deep connection Nigerian society shares with religious rites and the belief in metaphysical powers, the fact that it cannot be scientifically proven makes it unreliable [33].

\section{Interpretation and Assessment of Depression in Some Nigerian Communities}

Owing to the diversities in culture and traditions in Nigeria, clinicians and medical professionals have reported ethnic variances in reporting and diagnosing geriatric depression. [34] in his report of the nature and diagnosis of depressive disorders in Nigerian elderly highlighted differences in older adult's expression of depression based on cultural diversities, religion and social groupings. Over sixty percent of the Nigerian population will readily employ the services of traditional healers before consulting the conventional medical experts. This submission reported by [35] alluded to the general consensus that traditional or alternative medicine is widely perceived as a more affordable and accessible form of health care than the highly overrated conventional medicine. [36] argued the sensitivity of traditional health care services offered by traditionalists like sorcerers, witches, diviners etc. to the human emotional, environmental and spiritual consciousness. Their argument considered in some quarters to have been stretched beyond biological influences of medical practice, has unfortunately remained highly efficient and available in every society till date.

Postulations from the World Health Organization [37] about depression assuming a widespread status of highly acclaimed mental illness by 2020 invariably projecting depression as a fast-rising epidemic. [38] believed that cultural beliefs and societal values will dictate the behavioural trends, individual conceptualization, modes of treatment and pattern of recovery to be adopted by individuals towards depression and its consequences. This supposition is further buttressed by Kessing referenced in [39] whose definition of culture encompasses a cluster of ideas, laws and interpretations underlying human existence and interactions. His definition further expressed a clear understanding of individual's views of his surroundings, his expression of emotions towards events, occurrences, people around and beyond, towards higher beings, spiritual forces and his ability to maintain an equilibrium. Cultural studies across the world however presents a contrasting opinion on the influence of culture on the expression of depression. In their view, culture can be interpreted and accepted differently, in other words presenting the uniqueness of culture to different people across tribes and settlements. For instance, depressive mood in a community context in Nigeria is often ascribed to contravention of cultural interdictions, spiritual truculence, evil machinations, intrusion of objects, afflictions by god/sorcery, spiritual/ religious influence, family retribution, cultural traditional abuse, consequences of disobedience to traditional beliefs [36]. Community dwelling older adults may be limited in their need to seek help for mental health conditions that seemingly defy ordinary reasoning. There are however alternative traditional means employed over time and documented in literature to be traditionally effective in managing mental health related issues some of which are use of purgatives, making bodily incision, offering sacrifices/appeasing the Gods, fasting and prayers, organizing deliverance sessions, performing exorcisms etc. Comparatively, [40] reported that African traditionalists will report symptoms of geriatric depression as gloominess, head/lead formication, neuralgic sensations, bloating etc. Contrary to these beleifs, depressive symptoms as listed in [41] are indicative of consistent emotional rollercoaster, disappointment, loss of self-esteem and boredom. 


\section{Late Life Depression Management}

The disadvantage for adults diagnosed with depression might have been considered enormous due to conflicts in diagnoses, identification, management and treatment. Advocacy for proper management and treatment plans for depression implores clinicians to take into cognizance the patient's treatment of choice, anecdotal records, entry data, mental and physical health status in relation to age and socioeconomic status. Before initiating diagnosis, it behoves of clinicians to investigate patient's reactions to treatment models available and put into consideration their fear of medication and treatment reaction. Older adults need to be reassured about their worries of drug dependence and addiction. Contrary to the general misconceptions, antidepressants are not inhibitors. Obvious emotional reactions to a loss of a significant other, to poverty and wants cannot be suppressed by antidepressant or medication use [42]. Concerns and issues relating to depression management can be resolved successfully by a thorough and not selective listening, awareness and regular reassurance.

Available evidences indicated that geriatric depression can be effectively managed in and out of a clinical environment with psychotherapies, pharmacotherapies, electroconvulsive therapy (ECT) or more effectively psychotherapies can be combined with pharmacotherapies for enhanced results [43,44]. A general specification employed by geriatric psychiatrists globally proposed the use of the combination of drugs (antidepressants) with psychological interventions (CBT, reminiscence therapy, interpersonal therapy etc.) for effective outcome of a reduced depression [45]. There was an initial classification of antidepressant use as a standalone treatment regimen considered as another management option for depression [10]. Electroconvulsive therapy became another relative management option for chronic depressive disorders in the event of antidepressant use and psychotherapeutic failure or on occasion of the presence of suicidal intentions or life-threatening medical comorbidities. Psychotherapy is a talking treatment between the psychotherapist and a client i.e. the depressed individual. However, the topic and the treatment plan depend on the type of therapy being utilized.

\section{Pharmacotherapy}

In Nigeria, over twenty antidepressants (depression induced medications) have been authorized and certified safe by the National Agency for Food, Drug Administration and Control (NAFDAC) for depression in the elderly [46]. Antidepressants had been the official prescription for depression in most health facilities in Nigeria. Its wider acceptability is not void of complaints of side effects like excessive weight gain, dizzy spells, bowel irritation, anxiety etc. Although complaints of complications from the use of antidepressants in old age have been received in various quarters, complications of medications for geriatric depression treatment can be fueled by different factors which include multifarious use of medications more than the younger population, increased potential interactions of multiple prescriptions and age [47].

\section{Electroconvulsive Therapy}

The efficacy of the electroconvulsive therapy in a number of controlled studies on late life depression have been expressed in a percentage range of $60-80 \%[43,45]$. Electroconvulsive therapy is a psychological intervention that involves the passage of tiny voltage of electric current through to the brain triggering a short but reflexive seizure, the procedure is generally done under anesthesia. ECT is selectively suggested for patients with diagnosable resistant depression whose cases is considered a risk to self and people around. It is a form of psychotherapy that involves a complete alteration of the brain composition causing an immediate reverse of depressive symptoms. Treatment duration is usually between six to twelve weeks of hospital stay which in most cases is in a psychiatric facility. ECT intervention is not totally free of treatment side-effects, patients record complaints of mild to severe headache, delusion, temporary amnesia all of which persists for few days and responds to analgesics and corresponding medications. In severe cases of side effects, mortality rates for the utilization of ECT is minus $1 \%$ death in 10,000 depressed patients treated putting the ratio at 1:10 [43]. Experts thus advise that following each session of ECT, a maintenance medication should immediately be commenced to avert and put in check consequent relapse.

\section{Psychotherapy}

The talking treatment as it is referred to in some climes, psychotherapy is the process of engaging in an in-depth discussion (sessions) with a patient referred to as client to obtain facts and information regarding his current emotional state. Studies on depression in both young and older populations have established the effectiveness of psychotherapies as a treatment regimen. Its efficacy in resolving psychological disorders have established its empirical relevance across cultures [24]. Cognitive behavioral therapy, interpersonal psychotherapy, cognitive reminiscence therapy, problem-solving therapy are few empirically supported psychotherapies effective for managing geriatric depression. Other evidence-based therapies established to be adequately effective but not extensively explored for the management of geriatric depression carefully considering cognitive challenges, cardiac implications, limitations in function and physical illness in old age include supportive therapy, laughter therapy, psychodrama, music therapy, dance and movement therapy, humor therapy etc. Psychotherapies are structured discussion between a professional trained in the act and a client, psychotherapeutic sessions are usually between six to twelve sessions delivered within a period of six to eight weeks. Comparisons in the effectiveness of psychotherapies and pharmacotherapy cannot be effectively established in literature as about $45 \%-70 \%$ patients who underwent a therapeutic session recorded success rate that is quite similar to statistics observed across patients treated with antidepressants [48].

\section{Conclusion}

The sudden but precipitated increase in incidences of depression across all ages had necessitated the need for constant reviews of mental health disorders. Factors that informed the mental health decisions of community dwelling older adults are hinged on their personal convictions on the presence of the disease and not just another somatic related illness. These convictions are quite germane to the kind of treatment they sought for and could invariably be the major reason why geriatric depression has consistently been misdiagnosed or undertreated 
very often among community dwelling older adults. There is a constant basis for disagreement between mental health professionals, medical experts and psychotherapists alike on the construction of experience of depression especially by rural community dwellers. This is particularly relevant in Nigeria especially in communities surrounded with diverse cultures and beliefs confirming the age long belief in herbal mixtures and concussion, which seems to be appealing and realistic enough to be accepted as the immediate choice of health care. More studies exploring assessment, diagnosis and management of late life depression in rural community samples of older adults are therefore necessary to further establish their needs.

\section{Acknowledgments}

The author will like to acknowledge the University of Ibadan for the resources.

\section{References}

1. WHO (2017) Depression and other common mental disorders: Global Health Estimates. World Health Organization, Geneva 2.

2. United Nations World Population Ageing Highlights https://ww.un.org/development/ desa/pd/\#UN population. Accessed December, 2020.

3. Blazer DG (2003) Depression in late life: Review and commentary. Journal of Gerontology 58: 249-265. [crossref]

4. Morgan JH (2013) Late Life Depression and Counselling Agenda. Exploring Geriatric Logotheraphy as a Treatment Modality. International Journal of Psychological Resources 6: 94-101.

5. Fredrick JT, Steinman LE, Prohaska T, Unutzer J, Snowden M (2007) CommunityBased Treatment of Late life Depression: An Expert Panel-Informed Literature Review. American Journal of Preventive Medicine 33: 222-249. [crossref]

6. Allan CL, Ebmeier KP (2013) Review of treatment for late life Depression. Advances in Psychiatric Treatment 19: 302-309.

7. Thapa SB, Martinez P, Clausen T (2014) Depression and its Correlates in South Africa and Ghana among people aged 50 and above. Findings from the WHO study on Global Aging and Adult Health. Journal of Psychiatry 17: 1000167.

8. Damme AV, Declercq T, Lemey L, Tandt H, Petrovic M (2018) Late Life Depression: Issues for the General Practitioner. International Journal of General Medicine 11: 113120. [crossref]

9. Sun SM, Stewart E (2009) Health Locus of Control and Cholesterol Representations in Older Adults: Results of the FRACTION survey. Encephale 30: 331-341. [crossref]

10. Alexopoulos GS (2019) Mechanisms and Treatment of Late Life Depression. Translational Psychiatry 9: 188. [crossref]

11. Osimade OM (2010) Effectiveness of Laughter Therapy and Music Intervention in the Psychological Management of Geriatric Depression among Rural Community Dwelling Older Adults in Oyo State, Southwest Nigeria. Journal of Psychology and Psychotherapy 10: 376.

12. Elderly Suicide Prevention Network 2005 Depression. Available at: http://www. ausinet.com/factsheet/espn. Accessed August 8, 2020.

13. Blazer D (2005) Depression in late life: Review and Commentary. Journal of Gerontology: Medical Sciences 58: 249-265. [crossref]

14. Adeniyi OO (2013) Urbanization and Mental Health Problems in Nigeria: Implications for Counselling. International Review of Sociology 25: 43-54.

15. Onya O and Stanley P (2013) Risk Factors for Depressive Illness among Elderly Gopd Attendees at Upth, Port Harcourt, Nigeria. IOSR Journal of Dental and Medical Sciences 5: 77-86.

16. American Psychiatric Association. Diagnostic and Statistical Manual of Mental Disorders ( $5^{\text {th }}$ ed) 2013.

17. Mahony JM, Lippman J (2010) Older Age and The Underreporting of Depressive Symptoms. Journal of the American Geriatrics Society 43: 216-221.
18. Miller F, Paradis S, Housck M (2010) The Influence of Background Music on the Performance of the Mini Mental State Examination with Patients Diagnosed with Alzheimer's Disease. Journal of Music Therapy 3: 196-206. [crossref]

19. Frazini LM (2001) A Psychological Intervention Reduces Inflammatory Markers by Alleviating Depressive Symptoms: Secondary Analysis of a Randomized Controlled Trial. Psychosomatic Medical Journal 71: 715-724. [crossref]

20. Fanous L, Gardner A (2012) Neuroticism and Major Depression in Late Life: A Population Based Twin Study. Social Indicators Research 40: 285-298.

21. Moradipanah F, Mohammadi E, Mohammadil AZ (2009) Effect of Music on Anxiety, Stress and Depression Levels in Patients Undergoing Coronary Angiography. Eastern Mediterranean Health Journal 5: 639-647. [crossref]

22. Blackburn P, Wikins HM, Wiese B (2017) Depression in older adults: diagnosis and management. BCMJ 59: 171-177.

23. Shah A, Herbert R, Lewis S, Mahendran R, Platt J, et al. (2007) Screening for Depression among Acutely Ill Geriatric in-patients with a short Geriatric Depression Scale. Age and Aging 26: 217-221. [crossref]

24. Osimade OM (2020) Laughter Psychotherapy: An Adjunct to Clinical Management of Geriatric Depression among Rural Community Dwellers in Oyo State, Southwest Nigeria. Journal of Gerontology and Geriatric Research 9: 522.

25. Tisdale N (2010) Biological Risk actors of Late Life Depression. European Journal of Epidemiology 18: 745-750.

26. Isabella B, Henriette E (2008) The Relation between Depressive Symptoms and Age among older Europeans. Findings from SHARE. Vienna Institute of Demography: Working Papers.

27. Girma M, Hailu M, Wakwoya A, Yohannis M, Ebrahim J (2016) Geriatric Depression in Ethiopia: Prevalence and Associated Factors. Journal of Psychiatry 20: 1-5.

28. Robinson R, Price T (2013) Post-stroke depressive disorders: a follow-up study of 103 patients. Stroke. Journal of Mental Health 13: 635-641. [crossref]

29. Fiske A, O'Riley A, Widoe R (2008) Physical Health and Suicide in Late Life. Clinical Gerontology 31: 31-50.

30. National Survey on Drug Use and Health Report (2009) Suicidal Thoughts and Behaviour among adults. Available at http://oas.samhsa.gov/. Accessed: September, 2016

31. Wanyioke BW (2014) Depression as a Cause of Suicide. The Journal of Language, Technology and Entrepreneurship in Africa 5.

32. Jokotade M (2017) The more we talk about depression-induced suicide, the better.

33. Nwosu S, Odesanmi W (2001) Pattern of Suicide in Ile Ife, Nigeria. West African Journal of Medicine 20: 259-262. [crossref]

34. Morakinyo O (2002) The Nature and Diagnosis of Depressive Disorders in Africans In Morakinyo O. (ed) Handbook for students on Mental Health, Obafemi Awolowo University Teaching Hospital Complex.

35. Sloan R, Bagiella E, Powell T (1999) Religion, Spirituality, and Medicine. Lancet 353 664-667.

36. Yusuf A, Adeoye M (2012) Prevalence and Causes of Depression Among Civil Servants in Osun State: Implications for Counselling. Edo Journal of Counselling 4: 2.

37. WHO (2009) Pharmacological treatment of mental disorders in primary health care. World Health Organization. Geneval -68

38. Rose SR (2012) The Psychological Effects of Anxiolytic Music/Imagery on Anxiety and Depression Following Cardiac Surgery. PhD Thesis, Walden University, Minneapolis, MN 345-355.

39. Tjale AE (2004) Psychotherapy and Religious Values. Journal of Consulting and Clinical Psychology 48: 95-105.

40. Ayorinde O, Gureje O, Lawal R (2004) Psychiatric Research in Nigeria: Bridging Tradition and Modernization. British Journal of Psychiatry 184: 536-538. [crossref]

41. Busari AO (2007) Evaluating the Relationship between Gender, Age, Depression and Academic Performance among Adolescents. Scholarly Journal of Education 6-12.

42. Walker J (2011) Control and the Psychology of Health. City Open University Press Buckingham. 
43. Frazer C, Christenssen H, Griffiths KM (2005) Effectiveness of Treatments for Depression in Older people. Medical Journal Aust 182: 627- 632. [crossref]

44. Unutzer S, Katon W, Callahan CM, Williams JW, Hunkeler E, et al. (2002) Collaborative Management of Late Life Depression in the Primary Care Settings: A Randomized Controlled Trial. JAMA 12: 2836-2845.

45. Crawford M, Prince M, Menezes P, Mann A (2012) The Recognition and Treatment of Geriatric Depression in Primary Care. International Journal of Geriatric Psychiatry. Epidemiology 36: 613-620.
46. Nigerian Standard Treatment Guidelines. 2008. In Standard Treatment Guidelines (Nigeria) Nigerian Federal Ministry of Health in collaboration with the World Health Organization (WHO), EC, DFID.

47. George L, Social Factors, Depression, and Aging. In R. H. Binstock and L.K. George (Eds.), Handbook of Aging and the Social Sciences $7^{\text {th }}$ Edition 2011. 149-162. San Diego: Academic Press.

48. Kelly A, Zissleman I (2000) Combined Pharmacotherapy and Psychotherapy as Maintenance Treatment for Late-Life Depression: Effects on Social Adjustment. American Journal of Psychiatry 159: 466-468. 